\title{
ACTC1 as an invasion and prognosis marker in glioma
}

\author{
Shunya Ohtaki, MD, ${ }^{1,2}$ Masahiko Wanibuchi, MD, PhD, ${ }^{1,2}$ Yuko Kataoka-Sasaki, MD, PhD, ${ }^{2}$ \\ Masanori Sasaki, MD, PhD, ${ }^{2,3,4}$ Shinichi Oka, MD, PhD, ${ }^{2}$ Shouhei Noshiro, MD, ${ }^{1,2}$ \\ Yukinori Akiyama, MD, PhD, ${ }^{1}$ Takeshi Mikami, MD, PhD, ${ }^{1,2}$ Nobuhiro Mikuni, MD, PhD, ${ }^{1}$ \\ Jeffery D. Kocsis, PhD, ${ }^{3,4}$ and Osamu Honmou, MD, PhD2,3,4
}

\begin{abstract}
Departments of ${ }^{1}$ Neurosurgery and ${ }^{2}$ Neural Regenerative Medicine, Research Institute for Frontier Medicine, Sapporo Medical University School of Medicine, Sapporo, Hokkaido, Japan; ' ${ }^{3}$ Department of Neurology, Yale University School of Medicine, New Haven; and ${ }^{4}$ Center for Neuroscience and Regeneration Research, VA Connecticut Healthcare System, West Haven, Connecticut
\end{abstract}

OBJECTIVE Glioma is a major class of brain tumors, and glioblastoma (GBM) is the most aggressive and malignant type. The nature of tumor invasion makes surgical removal difficult, which results in remote recurrence. The present study focused on glioma invasion and investigated the expression of actin, alpha cardiac muscle 1 (ACTC1), which is 1 of 6 actin families implicated in cell motility.

METHODS mRNA expression of ACTC1 expression was analyzed using quantitative real-time polymerase chain reaction (qRT-PCR) in 47 formalin-fixed, paraffin-embedded glioma tissues that were graded according to WHO criteria: Grade I $(n=4)$; Grade II $(n=12)$; Grade III $(n=6)$; and Grade IV $(n=25)$. Survival was analyzed using the Kaplan-Meier method. The relationships between ACTC1 expression and clinical features such as radiological findings at the time of diagnosis and recurrence, patient age, Karnofsky Performance Scale status (KPS), and the MIB-1 index were evaluated.

RESULTS The incidence of ACTC1 expression as a qualitative assessment gradually increased according to WHO grade. The hazard ratio for the median overall survival $(\mathrm{mOS})$ of the patients with ACTC1-positive high-grade gliomas as compared with the ACTC1-negative group was $2.96(95 \% \mathrm{Cl}, 1.03-8.56)$. The mOS was 6.28 years in the ACTC1-negative group and 1.26 years in the positive group $(p=0.037)$. In GBM patients, the hazard ratio for mOS in the ACTC1-positive GBMs as compared with the ACTC1-negative group was $2.86(95 \% \mathrm{Cl} 0.97-8.45)$. mOS was 3.20 years for patients with ACTC1-negative GBMs and 1.08 years for patients with ACTC1-positive GBMs $(p=0.048)$. By the radiological findings, $42.9 \%$ of ACTC1-positive GBM patients demonstrated invasion toward the contralateral cerebral hemisphere at the time of diagnosis, although no invasion was observed in ACTC1-negative GBM patients $(p=0.013)$. The recurrence rate of GBM was $87.5 \%$ in the ACTC1-positive group; in contrast, none of the ACTC1-negative patients demonstrated distant recurrence (0.007). No remarkable relationship was demonstrated among ACTC1 expression and patient age, KPS, and the MIB-1 index.

CONCLUSIONS ACTC1 may serve as a novel independent prognostic and invasion marker in GBM.

http://thejns.org/doi/abs/10.3171/2016.1.JNS152075

KEY WORDS ACTC1; astrocytoma; cytoskeletal filament; glioblastoma; high-grade glioma; invasion; prognosis; oncology

$\mathrm{G}$ LIOBLastoma (GBM) displays an aggressive proliferation of tumor cells, and patients demonstrate very short survival despite treatments including surgical removal, irradiation, and chemotherapy. ${ }^{33}$ The 5-year survival rate of GBM patients is $4.7 \%$ to
$13.8 \% .^{9,24,32} \mathrm{GBM}$ patients frequently develop recurrence, not only locally but also in remote areas of brain. The infiltration of glioma cells, which constitutes a limiting factor of complete tumor removal, results in poor prognosis. The progression-free survival (PFS) and overall survival (OS)

ABBREVIATIONS ACTC1 = actin, alpha cardiac muscle 1; CE-T1 = contrast-enhanced T1-weighted; $\mathrm{Cl}=$ confidence interval; FFPE = formalin-fixed, paraffin-embedded; FLAIR = fluid-attenuated inversion recovery; GAPDH = glyceraldehyde 3-phosphate dehydrogenase; $G B M=$ glioblastoma; HGG = high-grade glioma; IDH = isocitrate dehydrogenase; KPS = Karnofsky Performance Scale; LGG = low-grade glioma; MGMT = O6-methylguanine-DNA methyltransferase; $\mathrm{mOS}=$ median overall survival; $\mathrm{mPFS}=$ median progression-free survival; OS = overall survival; PFS = progression-free survival; qRT-PCR = quantitative real-time polymerase chain reaction.

SUBMITTED September 2, 2015. ACCEPTED January 27, 2016.

INCLUDE WHEN CITING Published online April 15, 2016; DOI: 10.3171/2016.1.JNS152075. 
in patients with GBM can be quite variable with poor prediction of survival.

Glioma has 2 major characteristics: proliferation and infiltration. The former may be associated with local recurrence after incomplete tumor removal, and the latter is related to remote recurrence that is separate from the original tumor location. Patients with recurrence without infiltration resulting in local regrowth can be good candidates for surgical reexploration; however, patients with recurrence caused by the infiltration of tumor cells, which leads to remote recurrence especially at deep-seated locations or multiple areas, are not. The difficulty of tumor removal due to tumor infiltration has been strongly related to poor prognosis in glioma. . $^{17,27,39}$ Although the process of infiltration has been analyzed by molecular signatures such as matrix metalloproteinase, CD44, neural cell adhesion molecule, cadherin, integrin, and hepatocyte growth factor, the clinical relevance of these markers has not yet been fully studied. ${ }^{20,22,35}$ Elucidating the regulatory mechanisms of GBM infiltration holds promise for improving prognosis.

One of the causes of remote recurrence by glioma infiltration is considered enhancement of cell motility provided by the cytoskeletal filaments that consists of 3 major components: actin filaments, microtubules, and intermediate filaments. Among these cytoskeleton components, actin polymerization provides a transfer of intracellular materials and cell motility, including chemotaxis, mitogenesis, and path finding by axons. ${ }^{5,26,29}$ Actin dynamics-mediated cellular protrusion, which is often the first step in cell locomotion, is essential for cell invasion..$^{34}$ In this study, we focused on the glioma cell expression of actin, alpha cardiac muscle 1 (ACTC1) in various WHO-defined glioma grades in relation to prognosis and radiological studies in order to determine if ACTC1 may serve as a novel independent prognostic and invasion marker.

\section{Methods}

\section{Patients and Tissue}

Newly diagnosed consecutive patients between 2 and 80 years of age with diagnosed and histologically confirmed WHO Grade I to IV gliomas by several independent pathologists at our university hospital were eligible for this study. All tumors were maximally resected to preserve neurological function and followed with radiochemotherapy, as reported by Stupp et al. for high-grade gliomas (HGGs; i.e., Grade III and IV). ${ }^{33}$ The glioma tissues obtained in the operating room between November 2001 and October 2014 were fixed in liquid buffered formalin. A total of 47 formalin-fixed, paraffin-embedded (FFPE) tissues, including 4 WHO Grade I samples, 12 WHO Grade II samples, 6 WHO Grade III samples, and 25 WHO Grade IV samples, were analyzed. Written informed consent was obtained from each patient in accordance with the local ethics guidelines. This study was approved by the Institutional Review Board of Sapporo Medical University.

\section{Surveillance and Follow-Up}

OS and PFS were analyzed using the Kaplan-Meier sur- vival analysis. Tumor progression is defined by increasing tumor size, new areas of tumor, or unequivocal neurological deterioration. ${ }^{19}$ Since the oldest surgery of those cases was performed in 2001, this patient was followed up for 12.4 years.

\section{RNA Extraction, cDNA Synthesis, and Real-Time qRT-PCR}

FFPE samples were sectioned using a microtome and collected in 2-ml microtubes. RNA extraction was performed using deparaffinization solution (Qiagen) and the RNeasy FFPE Kit (Qiagen) according to the manufacturer's protocol. The total RNA concentration and A260/ A280 ratio were measured using a NanoDrop Lite spectrophotometer (Thermo Fisher Scientific Inc.). Samples in which the A260/A280 ratio was less than 1.8 were excluded. Samples with an RNA concentration less than 40 $\mathrm{ng} / \mu \mathrm{l}$ were prepared using a centrifugal concentrator. The QuantiTect Reverse Transcription Kit (Qiagen) was used to reverse transcribe $500 \mathrm{ng}$ of total RNA. PCR reactions, which were $10 \mu \mathrm{l}$ in volume, were prepared with $2 \mu \mathrm{l}$ cDNA diluted to 1:5. TaqMan Universal Master Mix II with UNG and TaqMan Gene Expression assays for glyceraldehyde 3-phosphate dehydrogenase (GAPDH; Hs02758991_g1) and ACTC1 (Hs00606316_ml) were purchased from Thermo Fisher Scientific Inc. Quantitative real-time polymerase chain reaction (qRT-PCR) was performed in quintuple using PRISM7500 (Thermo Fisher Scientific Inc.), and the entire experiment was repeated twice. The presence of ACTC1 on qRT-PCR was the criterion for defining a sample as ACTC1 positive, and the absence of ACTC1 classified a sample as negative. The thermal cycling parameters were $50^{\circ} \mathrm{C}$ for 2 minutes and $95^{\circ} \mathrm{C}$ for $10 \mathrm{~min}$ utes, followed by 60 cycles of $95^{\circ} \mathrm{C}$ for 15 seconds and $60^{\circ} \mathrm{C}$ for 1 minutes. The cycle threshold value of each gene of interest was calculated with reference to the GAPDH value. After obtaining written informed consent, the fold change was determined using the $2^{-\mathrm{ddCt}}$ method relative to the calibrator, which was obtained during surgery with internal and external decompression because of trauma. ${ }^{3}$

\section{Immunohistochemical Analysis}

The FFPE blocks were sectioned into slides with a thickness of $3 \mu \mathrm{m}$ and then deparaffinized. Sections were immunostained using BGX-Ki 67 (BioGenex) according to the manufacturer's instructions with the anti-Ki 67 monoclonal antibody. The MIB-1 index was calculated as the percentage of positively stained tumor cell nuclei in areas with the greatest degree of immunostaining. ${ }^{28}$

\section{Radiological Evaluation}

Radiological analysis was performed by 2 neuroradiologists who were independent of this study. A tumor that extended beyond the corpus callosum toward the direction of the contralateral hemisphere was judged as invasive, especially at the time of diagnosis. A tumor that occurred at another location without direct connection with the cavity after tumor removal was judged as recurrence.

\section{Statistical Analysis}

Data were statistically analyzed, and they are presented 
as the mean values \pm the standard error of the mean. Differences among groups were assessed using the KruskalWallis test, and the Mann-Whitney U-test and Spearman test were used to identify group differences. All statistical analyses were performed using SPSS (version 22) (IBM Corp.). Differences were deemed statistically significant if $\mathrm{p}<0.05$. Comparison of OS and PFS according to ACTC1 expression was performed with the log-rank test.

\section{Results}

\section{Expression Of ACTC1 in Low-Grade Gliomas and HGGs}

Qualitative assessment of the positivity rate of ACTC1 using real-time qRT-PCR indicated an increase in HGGs as compared with low-grade gliomas (LGGs: Grades I and II). The incidence was $31.3 \%$ for LGGs and $58.1 \%$ for HGGs ( $\mathrm{p}=0.081)$ (Fig. 1A). The expression of ACTC1 mRNA as a quantitative assessment was significantly elevated in HGGs ( $\mathrm{p}=0.024)$ (Fig. 1B).

\section{Survival and Progression}

The OS and PFS for patients with Grade II to IV gliomas and only Grade IV gliomas are shown in Fig. 2 and Table 1 . For the HGG group, the median survival benefit in the ACTC1-negative group was 5.02 years; the median OS (mOS) was 6.28 years in ACTC1-negative HGG patients and 1.26 years in ACTC1-positive HGG patients ( $\mathrm{p}$ $=0.037$ ) (Table 1 and Fig. 2A). The hazard ratio, which was adjusted by fitting the Cox proportional-hazard models for mOS in the ACTC1-positive HGG patients as compared with the ACTC1-negative patients, was $2.96(95 \%$ CI 1.03-8.56). These data indicate 5 times longer OS in ACTC1-negative HGG patients compared with ACTC1positive patients. The median PFS (mPFS) benefit in the ACTC1-negative group was 1.63 years; the mPFS was 2.08 years in ACTC1-negative HGG patients and 0.50 years in ACTC1-positive HGG patients $(\mathrm{p}=0.032)$. The hazard ra- tio for mPFS was 2.54 (95\% CI 1.10-5.83) (Table 1 and Fig. 2B).

The mOS for the Grade IV GBM patients was 3.20 years in ACTC1-negative GBM patients and 1.08 years in ACTC1-positive GBM patients $(\mathrm{p}=0.048)$, indicating a median survival benefit in the ACTC1-negative group of 2.12 years (Table 1 and Fig. 2C). The hazard ratio for mOS in the ACTC1-positive GBM patients as compared with the ACTC1-negative patients was 2.86 (95\% CI 0.97-8.45). These data indicate about 3-times longer overall survival in ACTC1-negative GBM patients compared with ACTC1positive patients. The mPFS benefit in the ACTC1-negative group was 0.69 years; mPFS was 1.13 years in ACTC1negative GBM patients and 0.44 years in ACTC1-positive GBM patients $(\mathrm{p}=0.020)$. The hazard ratio for $\mathrm{mPFS}$ was 2.82 (95\% CI 1.14-6.98) (Table 1 and Fig. 2D). Thus, both OS and PFS were prolonged in the ACTC1-negative groups in patients with Grade II to IV, Grade III and IV, and Grade IV only gliomas.

\section{Radiological Findings at the Time of Diagnosis in GBMs}

As for invasion, $42.9 \%$ of tumors demonstrated invasion toward the contralateral cerebral hemisphere in ACTC1positive GBM patients, although none of the instances of invasion were observed in ACTC1-negative GBM patients $(\mathrm{p}=0.013)$. The finding of deep-seated invasion to the contralateral cerebral hemisphere was observed in ACTC1positive GBMs at the time of diagnosis. The typical invasion pattern was progression through the corpus callosum. Frontal tumors demonstrated progression through the genu and the anterior part of the body of the corpus callosum (Fig. 3A and B), and occipital tumors spread through the splenium of the corpus callosum (Fig. 3C). In contrast, the ACTC1-negative GBMs indicated topographical unity near the surface of the cortex. Even though the maximum diameter of the ACTC1-negative tumors was $62 \mathrm{~mm}$, no invasion was observed (Fig. 3D). Two other patients with ACTC1-negative tumors revealed similar radiological findings (Fig. 3E and F, respectively).
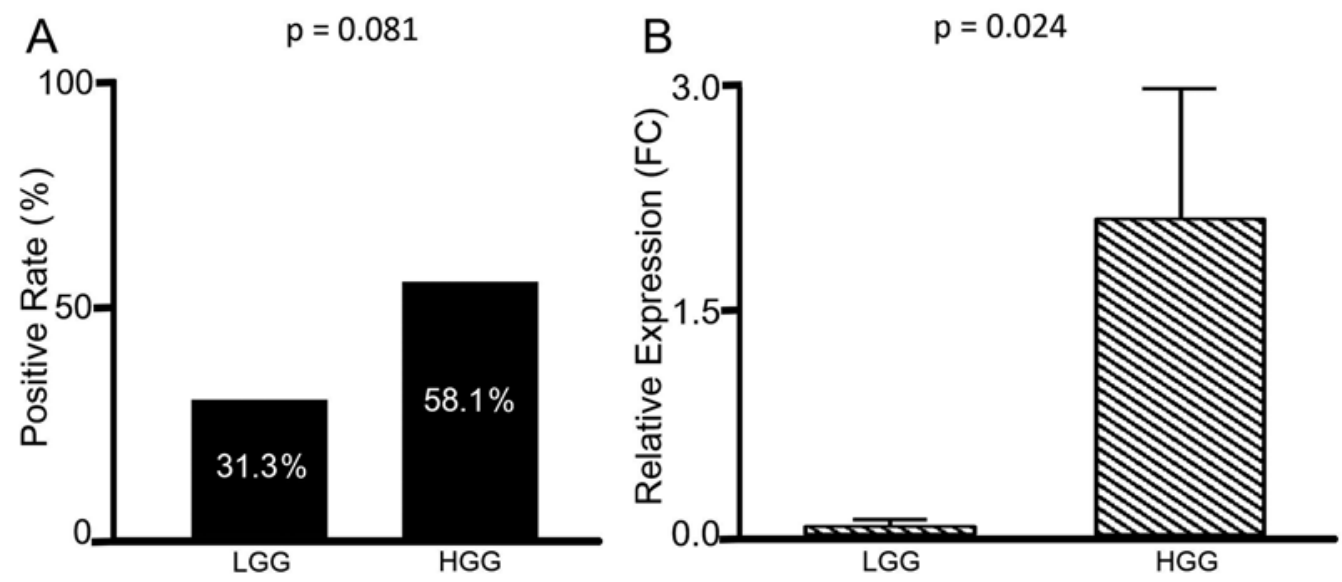

FIG. 1. Positivity rate and expression of ACTC1. mRNA expression for ACTC1 was analyzed in 47 FFPE glioma samples with WHO-defined grades: Grade I+II is LGG $(n=16)$, and Grade III+IV is HGG $(n=31)$. The results are expressed as the relative mRNA expression with respect to the WHO grade. A: The positivity rate of ACTC1 in HGGs was higher than in LGGs ( $p=$ 0.081). B: The expression of ACTC1 mRNA as a quantitative assessment was significantly elevated in HGGs as compared with LGGs $(p=0.024)$. The error bars indicate the standard error of the mean. $F C=$ fold change. 
TABLE 1. OS and PFS of ACTC1-negative and -positive gliomas*

\begin{tabular}{cccccccc}
\hline & \multicolumn{2}{c}{ ACTC1 } & & & & & \\
Variable & Negative (yrs) & Positive (yrs) & & $p$ Value & Hazard Ratio & $95 \% \mathrm{Cl}$ & Benefit (yrs) \\
\hline Median OS & & & & & & & \\
\hline Grade III+IV & 6.28 & 1.26 & 0.037 & 2.96 & $1.03-8.56$ & 5.02 \\
\hline Grade IV & 3.20 & 1.08 & 0.048 & 2.86 & $0.97-8.45$ & 2.12 \\
\hline Median PFS & & & & & & \\
\hline Grade III+IV & 2.08 & 0.50 & 0.032 & 2.54 & $1.10-5.83$ & 1.63 \\
\hline Grade IV & 1.13 & 0.44 & 0.020 & 2.82 & $1.14-6.98$ & 0.69 \\
\hline
\end{tabular}

* A total of 18 patients with ACTC1-positive HGG (Grade III and IV) and 13 patients with ACTC1-negative gliomas were compared. Among these, 14 patients with ACTC1-positive GBMs and 11 patients with ACTC1-negative GBMs were further investigated.

\section{Radiological Findings at the Time of Recurrence in GBMs}

Among recurrent GBM cases, $87.5 \%$ of ACTC1-positive tumors demonstrated remote recurrence, but no remote recurrence was observed in ACTC1-negative patients $(\mathrm{p}$ $=0.007)$. A representative case of ACTC1-positive GBM originating from the left putamen revealed heterogeneous ring enhancement without apparent perifocal edema (Fig. $4 \mathrm{~A}$ and $\mathrm{B}$ ). Recurrence was observed in the right temporal lobe at 20.3 months after the initial surgery followed by chemoradiotherapy (Fig. 4C and D). The recurrent lesion was in the contralateral side with presumed tumor spread through the commissural tracts. A case of an ACTC1negative GBM tumor, which was initially a single lesion, occurred at the temporal lobe with perifocal edema (Fig.
$4 \mathrm{E}$ and $\mathrm{F}$ ). Because the tumor was confined to a superficial location, gross-total removal of the enhanced tumor was accomplished. At 13.3 months after initial treatment, only local recurrence without any invasion toward multiple lobules or remote recurrence was observed (Fig. 4G and H).

Analysis of the HGGs indicated a $90.9 \%$ rate of remote recurrence in the ACTC1-positive group. In contrast, none of the ACTC1-negative patients demonstrated distant recurrence ( $\mathrm{p}=0.002$; Pearson's chi-square test). Among the observed recurrent cases, $21.4 \%$ were of Grade III gliomas, and all ACTC1-positive tumors demonstrated the remote recurrence of Grade III tumors. In contrast, all ACTC1-negative Grade III tumors remained localized to the initial tumor site.
Overall Survival

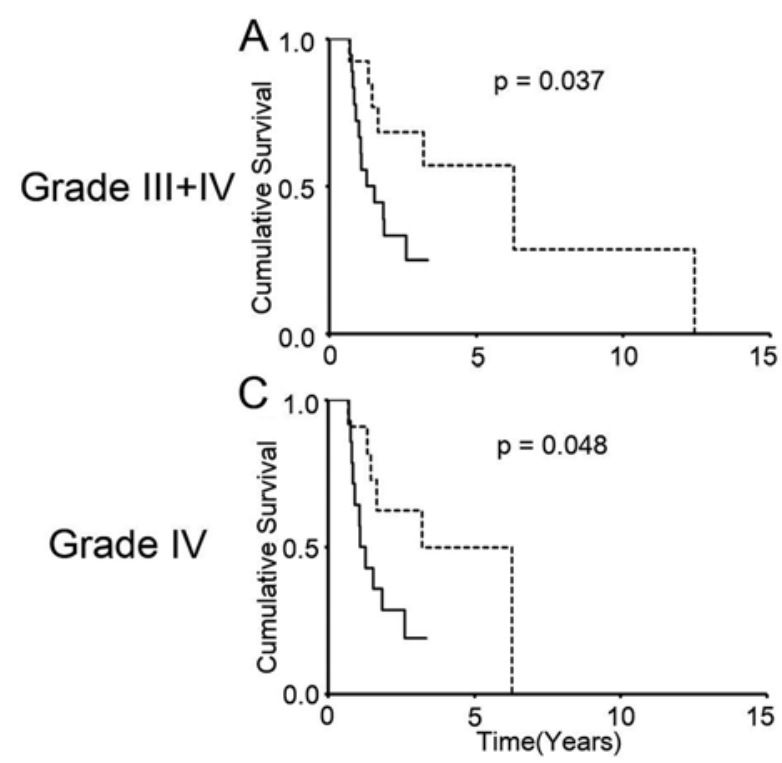

Progression Free Survival
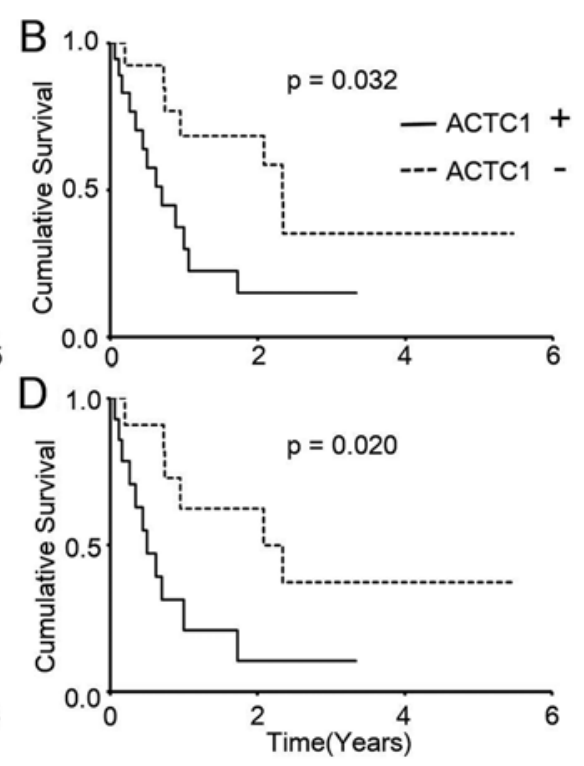

FIG. 2. Kaplan-Meier estimates of OS and PFS in the patients who had gliomas with the presence or absence of ACTC1 expression. A: The median survival of the HGG group (Grade III+IV) was 6.28 years for the 13 patients with ACTC1-negative HGG compared with 1.26 years for the 18 patients with ACTC1-positive HGG $(p=0.037)$. B: mPFS was 2.08 years for ACTC1-negative HGG patients and 0.50 years for ACTC1-positive HGG patients $(p=0.032)$. The median survival was 1.08 years for the 14 patients with ACTC1-positive GBMs compared with 3.20 years for the 11 patients with ACTC1-negative GBMs. C: The survival benefit in the ACTC1-negative group was 2.12 years $(p=0.048)$. D: The mPFS was 1.13 years for ACTC1-negative GBM patients and 0.44 years for ACTC1-positive GBM patients $(p=0.020)$. The benefit of mPFS in the ACTC1-negative group was 0.69 years. Survival distributions were compared with the use of the log-rank test. 


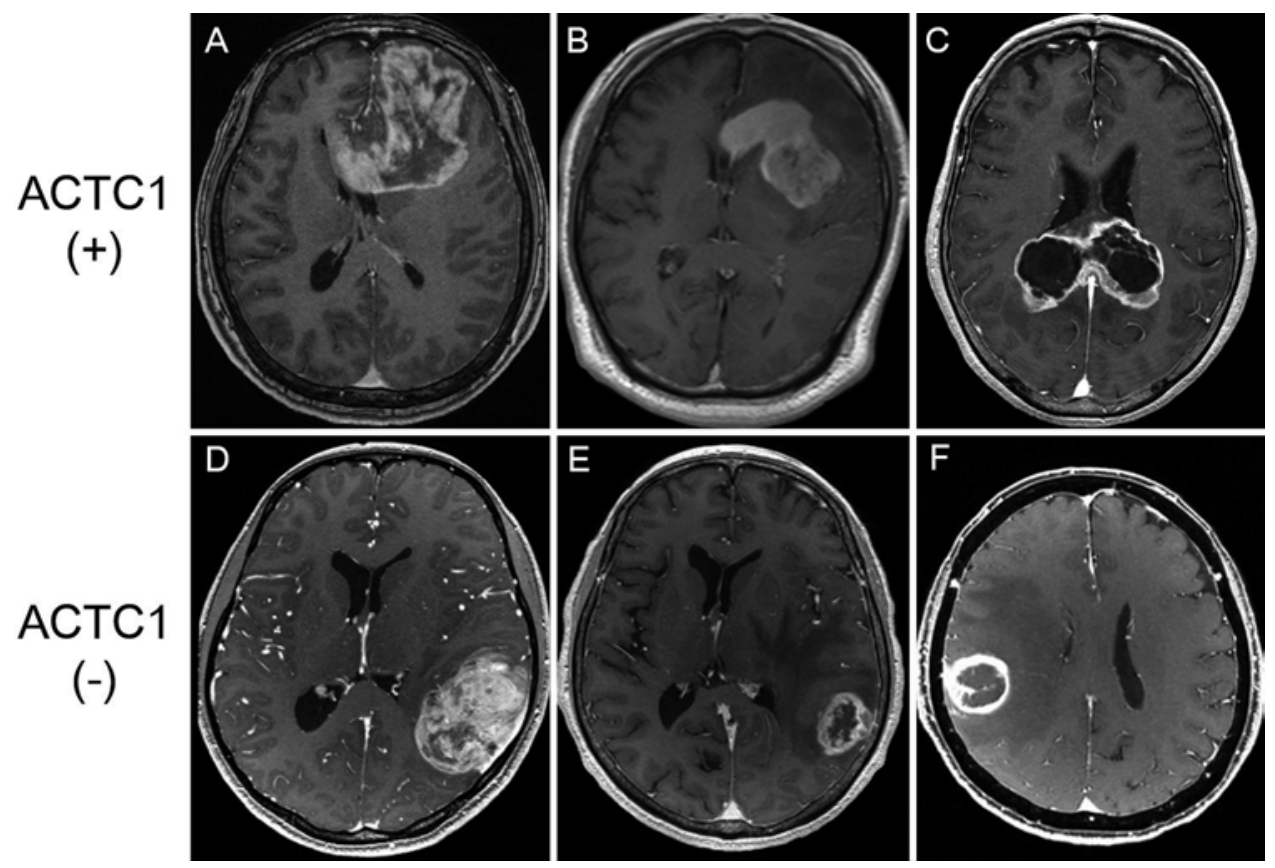

FIG. 3. MRI findings at the time of diagnosis. A and B: Findings obtained in patients with ACTC1-positive GBMs. A 60-year-old woman presented with symptoms of headache and nausea (A) and a 53-year-old man presented with symptoms of seizure (B). Both patients demonstrated tumor invasion toward the contralateral cerebral hemisphere through the genu and anterior third of the corpus callosum. C: Image obtained in a 71-year-old woman with an ACTC1-positive GBM who presented with headache, showing invasion of the GBM through the splenium of the corpus callosum. D-F: In contrast, ACTC1-negative GBMs developed at localized areas near the surface of the cortex and were associated with focal neurological deficits. A 19-year-old woman presented with symptoms of progressive sensory aphasia (D), a 63-year-old woman presented with sensory aphasia (E), and a 61-year-old woman presented with left hemiparesis.

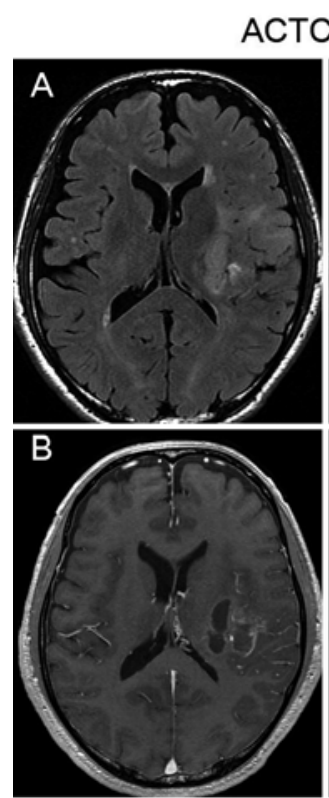

Initial
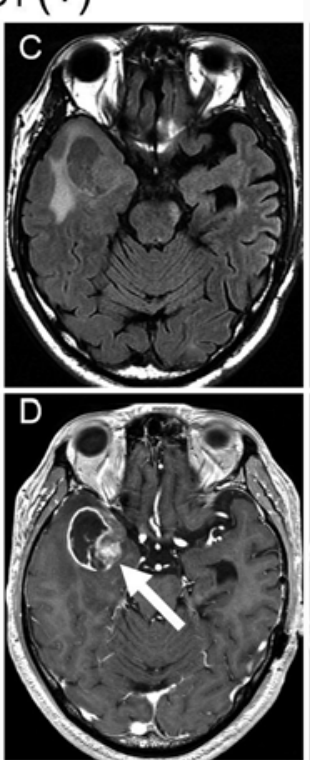

Recurrence

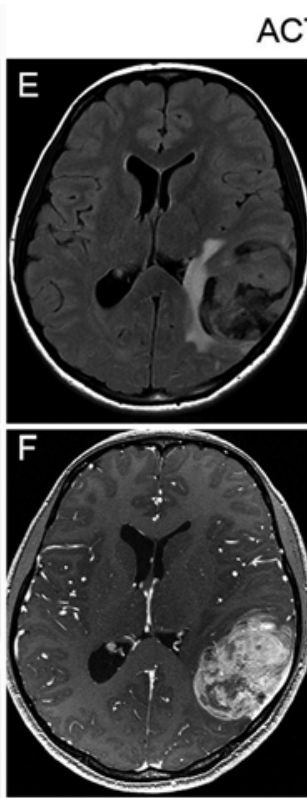

Initial
$\operatorname{ACTC1}(-)$

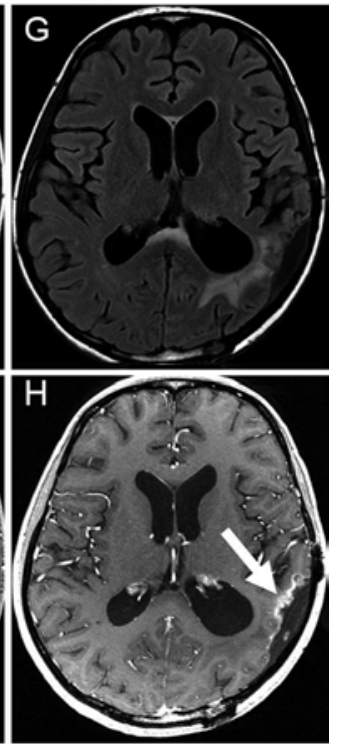

Recurrence

FIG. 4. MRI findings at the time of recurrence. A-D: A 57-year-old man with an ACTC1-positive GBM presented with left sensory disturbance at the time of diagnosis. FLAIR (A) and contrast-enhanced T1-weighted MR (B) images showed that the tumor originated from the left putamen with heterogeneous ring enhancement. Recurrence was observed in the right temporal lobe on FLAIR (C) and contrast-enhanced T1-weighted (D) images at 20.3 months after initial surgery followed by chemoradiotherapy. E-H: A 19-year-old woman with a ACTC1-negative GBM presented with progressive sensory aphasia. FLAIR (E) and contrast-enhanced T1-weighted $(F)$ images indicated that the tumor was localized at the posterior temporal lobe with perifocal edema. At 13.3 months after initial treatment, only local recurrence without any invasion toward multiple lobules or remote recurrence was observed on FLAIR (G) and contrast-enhanced T1-weighted images. 

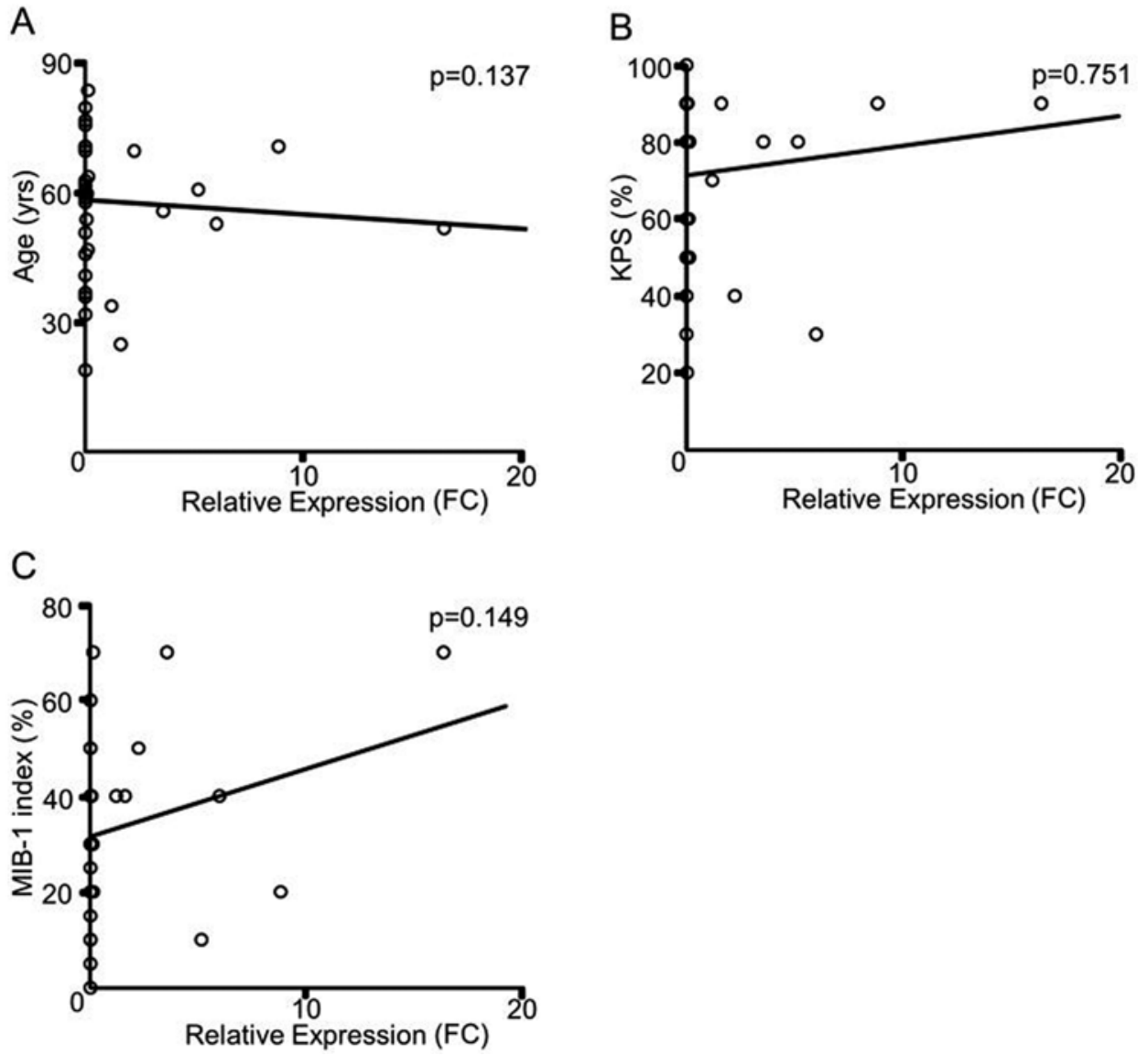

FIG. 5. Scatter plot analysis of ACTC1 mRNA expression with respect to patient age, KPS, and the MIB-1 index. No relationship was determined among ACTC1 mRNA expression and patient age (A), KPS (B), or the MIB-1 index (C).

\section{Relationship Among ACTC1 Expression and MIB-1 Index, Patient Age, and Karnofsky Performance Scale Status}

No relationship was observed between ACTC1 expression and patient age (Fig. 5A), Karnofsky Performance Scale status (KPS) (Fig. 5B), and MIB-1 index (Fig. 5C). Because both KPS and patient age are considered to have a relationship with the prognosis of GBM, ACTC1 expression may be an independent marker of glioma prognosis, apart from the KPS score and patient age. Since the MIB-1 index is commonly used as a marker of proliferation, the lack of a relationship between ACTC1 expression and the MIB-1 index suggests that ACTC1 expression differs from proliferation markers.

\section{Discussion}

We discovered that ACTC1 is expressed in a subset of glioma cases with a progressive increase from low to high grade (WHO Grade I-IV). In addition, the OS and PFS of patients who had ACTC1-positive tumors were remarkably poor when compared with ACTC1-negative tumors. The mOS of our GBM sample (both ACTC1-positive and -negative tumors) is 18.36 months, which is consistent with previous studies.,18,19 The mOS of GBM patients treated by surgical removal and radiotherapy was 12.1 months.,18,19 Survival was extended to 14.6 months by adding temozolo- mide. ${ }^{33}$ With a survival benefit of 2.5 months, temozolomide treatment became widely used as a standard chemotherapy agent for GBM..$^{33}$ mOS was extended to 16.7 months in a Phase III study (AVAglio). ${ }^{8}$ Among our GBM patients, the mOS of ACTC1-positive GBMs was 13.0 months, whereas that of ACTC1-negative patients was 38.4 months. Thus, ACTC1-positive GBM patients indicated much poorer survival than ACTC1-negative GBM patients. The survival benefit of 25.4 months in ACTC1-negative GBM patients was much longer than the benefit obtained by chemotherapy. Since survival was statistically different in the absence or presence of ACTC1 expression, ACTC1 may be considered a prognostic factor. In clinical studies that explore new therapeutic approaches for GBM, analysis of ACTC1-positive and -negative tumors may be informative.

Many genetic factors, such as phosphatase and tensin homolog deletion on chromosome 10, epidermal growth factor receptor, and tumor protein $\mathrm{p} 53$, were reported as prognostic factors. Among those, isocitrate dehydrogenase (IDH) mutation and methylation of the promotor of $\mathrm{O}^{6}$-methylguanine-DNA methyltransferase (MGMT) have been given heavy prognostic weight. However, the methylation of MGMT promotor identified in $45 \%$ to $75 \%$ of GBM tumors is strictly considered as a predictive factor for the treatment by temozolomide, not a prognostic fac- 
tor. ${ }^{2,13,15,23}$ Mutation of IDH is the most commonly used factor for the prognosis of HGGs. IDH mutation is considered to be a favorable factor in HGGs. ${ }^{12,25,37,38}$ For patients with GBM, the mOS was 31 months for mutated IDH as compared with 15 months for patients with wild-type IDH. The survival benefit in the presence of IDH mutation was 16 months. ${ }^{38}$ The survival benefit of 25.4 months by ACTC1negative GBMs was much longer than the benefit obtained by the mutation of IDH.

In our study, the radiological findings of the ACTC1positive GBM patients revealed tumor invasion toward the contralateral hemisphere at the time of initial diagnosis and remote recurrence at the time of recurrence. The invasion and remote recurrence of ACTC1-positive GBM cells in HGGs occurred through the commissural, association, or other projection fibers. ${ }^{1}$ Actin relates to cell motility in normal cells and invasion or metastasis in cancer cells. ${ }^{26}$ Actin is present in the cytoplasm just inside of the cell membrane. In the normal state, actin regulates cell motility and shape by repeating the expansion of the filaments by polymerization and contraction of the filaments by depolymerization. ${ }^{11,21,31}$ Thus, the expression of ACTC1 in GBM cells may be mechanistically related to their greater invasiveness than ACTC1-negative cells.

No clear relationship was determined between ACTC1 expression and KPS score or patient age. Prognostic factors have been associated with age, performance status, degree of surgical removal of the tumor, and postsurgical treatment. Favorable factors were young age, good KPS prior to surgery, and maximum tumor removal, 4,7,14,16,30,36 which were associated with a good response to chemotherapy and irradiation. Since ACTC1 expression has no relationship with the previously mentioned prognostic factors, ACTC1 may be considered an independent prognostic factor.

No relationship was observed between ACTC1 expression and the MIB-1 index, which detects the Ki-67 antigen. Ki-67, which was initially detected as an autoantibody in the blood of leukemia patients in 1992, is expressed during the $G_{1}, S, G_{2}$, and $M$ stages of the cell cycle. ${ }^{6}$ Since Ki-67 was detected inside the nucleus of active cells, the MIB-1 antibody, which detects $\mathrm{Ki}-67$, is considered a marker of proliferation. The lack of an association between ACTC1 and the $\mathrm{Ki}-67$ index suggests that the poor prognosis of ACTC1-positive GBM patients is due to higher tumor cell invasion along the nerve tracts, not an increase in cell proliferation. Thus, ACTC1 may represent an invasion marker that is independent of cell proliferation.

Our study revealed that the remote recurrence rate of ACTC1-positive HGGs was high (87.5\% for GBM only vs 90.9\% for HGGs); however, ACTC1-negative HGGs did not display remote recurrence, and their recurrence occurred in the primary (local) site. Although HGGs (especially GBMs) have a nature of recurrence, at present there is no way to predict if recurrence will occur only locally or at a remote site. Evaluating ACTC1 expression in $\mathrm{HGG}$ tissue may be beneficial for predicting remote recurrence.

Because ACTC1-positive GBM cells, which have a strong capacity to migrate, could exist in high-intensity areas on T2-weighted or fluid-attenuated inversion recovery (FLAIR) MRI images, supratotal resection including the high-intensity areas on T2-weighted or FLAIR imaging is encouraged..$^{10}$ Although the molecular mechanisms for migration should be elucidated in a future study, it is conceivable that ACTC1 might play an important role in the migratory capacity of GBMs.

In addition, the fact that ACTC1-negative GBMs recur locally and not in remote sites could encourage performing multiple surgeries in order to remove recurrent tumors because the ACTC1-negative GBMs may not display remote recurrence. The local control of tumors could be effective for extending mOS. Thus, ACTC1 might be an important molecule for molecular-based surgical decisions for GBM, in the context of a new suggested grading system based on biological molecular markers. ${ }^{18}$

Our study, which used FFPE glioma samples, has some advantages. The first is that the extensive retrospective analysis with long-term, clinical follow-up data could be achieved. The second is that the quantitative analysis by qRT-PCR of the mRNA expression associated with glioma-specific biomarkers, such as ACTC1 in this study, could be performed with small samples of FFPE tissue materials, although protein expression could not be measured. The third is that this research approach using FFPE tissue materials can be implemented at multiple institutions, not limited facilities, because it is easy to collect FFPE sample tissues from multiple institutions. Thus, this study approach using FFPE glioma materials is considered to be a simple and effective method for analyzing the relationship among the expression of $\mathrm{ACTC} 1$ and various clinical factors.

\section{Conclusions}

ACTC1 is expressed in a large subset of GBM tumors, especially high-grade tumors. ACTC1-positive GBMs indicated poorer prognosis compared with ACTC1-negative GBMs. The results of this study shed new light on the potential mechanisms of GBM invasion. ACTC1 may serve as a novel independent prognostic biomarker and a potential therapeutic target for molecular-based surgery for HGGs.

\section{Acknowledgments}

This work was supported, in part, by grants from the Japanese Ministry of Education, Culture, Sports, Science, and Technology and a Grant-in-Aid for Scientific Research (no. 26462187) awarded to Dr. Wanibuchi. Dr. Kocsis was supported, in part, by a Senior Research Career Scientist Award from the Rehabilitation Research and Development Service of the Department of Veterans Affairs (nos. B7335R and B9260L).

\section{References}

1. Bellail AC, Hunter SB, Brat DJ, Tan C, Van Meir EG: Microregional extracellular matrix heterogeneity in brain modulates glioma cell invasion. Int J Biochem Cell Biol 36:1046-1069, 2004

2. Bello MJ, Alonso ME, Amiñoso C, Anselmo NP, Arjona D, Gonzalez-Gomez P, et al: Hypermethylation of the DNA repair gene MGMT: association with TP53 G:C to A:T transitions in a series of 469 nervous system tumors. Mutat Res 554:23-32, 2004 
3. Bookout AL, Cummins CL, Mangelsdorf DJ, Pesola JM, Kramer MF: High-throughput real-time quantitative reverse transcription PCR. Curr Protoc Mol Biol Chapter 15:Unit 15.8, 2006

4. Bredel M, Piribauer M, Marosi C, Birner P, Gatterbauer B, Fischer I, et al: High expression of DNA topoisomerase II $\alpha$ and $\mathrm{Ki}-67$ antigen is associated with prolonged survival in glioblastoma patients. Eur J Cancer 38:1343-1347, 2002

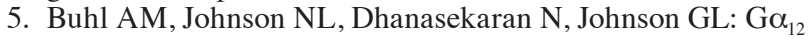
and $\mathrm{G \alpha}_{13}$ stimulate Rho-dependent stress fiber formation and focal adhesion assembly. J Biol Chem 270:24631-24634, 1995

6. Cattoretti G, Becker MH, Key G, Duchrow M, Schlüter C, Galle J, et al: Monoclonal antibodies against recombinant parts of the Ki-67 antigen (MIB 1 and MIB 3) detect proliferating cells in microwave-processed formalin-fixed paraffin sections. J Pathol 168:357-363, 1992

7. Chaichana KL, Jusue-Torres I, Navarro-Ramirez R, Raza SM, Pascual-Gallego M, Ibrahim A, et al: Establishing percent resection and residual volume thresholds affecting survival and recurrence for patients with newly diagnosed intracranial glioblastoma. Neuro Oncol 16:113-122, 2014

8. Chinot OL, de La Motte Rouge T, Moore N, Zeaiter A, Das A, Phillips H, et al: AVAglio: Phase 3 trial of bevacizumab plus temozolomide and radiotherapy in newly diagnosed glioblastoma multiforme. Adv Ther 28:334-340, 2011

9. Dolecek TA, Propp JM, Stroup NE, Kruchko C: CBTRUS statistical report: primary brain and central nervous system tumors diagnosed in the United States in 2005-2009. Neuro Oncol 14 (Suppl 5):v1-v49, 2012

10. Duffau H: Is supratotal resection of glioblastoma in noneloquent areas possible? World Neurosurg 82:e101e103, 2014

11. Halpain S: Actin and the agile spine: how and why do dendritic spines dance? Trends Neurosci 23:141-146, 2000

12. Hartmann C, Hentschel B, Wick W, Capper D, Felsberg J, Simon M, et al: Patients with IDH1 wild type anaplastic astrocytomas exhibit worse prognosis than IDH1-mutated glioblastomas, and IDH1 mutation status accounts for the unfavorable prognostic effect of higher age: implications for classification of gliomas. Acta Neuropathol 120:707-718, 2010

13. Hegi ME, Diserens AC, Gorlia T, Hamou MF, de Tribolet N, Weller M, et al: MGMT gene silencing and benefit from temozolomide in glioblastoma. N Engl J Med 352:997-1003, 2005

14. Houillier C, Lejeune J, Benouaich-Amiel A, Laigle-Donadey F, Criniere E, Mokhtari K, et al: Prognostic impact of molecular markers in a series of 220 primary glioblastomas. Cancer 106:2218-2223, 2006

15. Kamiryo T, Tada K, Shiraishi S, Shinojima N, Kochi M, Ushio Y: Correlation between promoter hypermethylation of the O6-methylguanine-deoxyribonucleic acid methyltransferase gene and prognosis in patients with high-grade astrocytic tumors treated with surgery, radiotherapy, and 1-(4-amino-2-methyl-5-pyrimidinyl) methyl-3-(2-chloroethyl)-3-nitrosourea-based chemotherapy. Neurosurgery 54:349-357, 2004

16. Krex D, Klink B, Hartmann C, von Deimling A, Pietsch T, Simon M, et al: Long-term survival with glioblastoma multiforme. Brain 130:2596-2606, 2007

17. Liu X, Wang X, Du W, Chen L, Wang G, Cui Y, et al: Suppressor of fused (Sufu) represses Gli1 transcription and nuclear accumulation, inhibits glioma cell proliferation, invasion and vasculogenic mimicry, improving glioma chemo-sensitivity and prognosis. Oncotarget 5:11681-11694, 2014

18. Louis DN, Perry A, Burger P, Ellison DW, Reifenberger G, von Deimling A, et al: International Society Of
Neuropathology-Haarlem consensus guidelines for nervous system tumor classification and grading. Brain Pathol 24:429-435, 2014

19. Macdonald DR, Cascino TL, Schold SC Jr, Cairncross JG: Response criteria for phase II studies of supratentorial malignant glioma. J Clin Oncol 8:1277-1280, 1990

20. Maher EA, Furnari FB, Bachoo RM, Rowitch DH, Louis DN, Cavenee WK, et al: Malignant glioma: genetics and biology of a grave matter. Genes Dev 15:1311-1333, 2001

21. Matus A: Actin-based plasticity in dendritic spines. Science 290:754-758, 2000

22. Nakada M, Nakada S, Demuth T, Tran NL, Hoelzinger DB, Berens ME: Molecular targets of glioma invasion. Cell Mol Life Sci 64:458-478, 2007

23. Nakamura M, Watanabe T, Yonekawa Y, Kleihues P, Ohgaki H: Promoter methylation of the DNA repair gene MGMT in astrocytomas is frequently associated with $\mathrm{G}: \mathrm{C}$ --> A:T mutations of the TP53 tumor suppressor gene. Carcinogenesis 22:1715-1719, 2001

24. Narita Y, Shibui S: Trends and outcomes in the treatment of gliomas based on data during 2001-2004 from the Brain Tumor Registry of Japan. Neurol Med Chir (Tokyo) 55:286-295, 2015

25. Nobusawa S, Watanabe T, Kleihues P, Ohgaki H: IDH1 mutations as molecular signature and predictive factor of secondary glioblastomas. Clin Cancer Res 15:6002-6007, 2009

26. Pollard TD, Cooper JA: Actin, a central player in cell shape and movement. Science 326:1208-1212, 2009

27. Que T, Song Y, Liu Z, Zheng S, Long H, Li Z, et al: Decreased miRNA-637 is an unfavorable prognosis marker and promotes glioma cell growth, migration and invasion via direct targeting Akt1. Oncogene 34:4952-4963, 2015

28. Ralte AM, Sharma MC, Karak AK, Mehta VS, Sarkar C: Clinicopathological features, MIB-1 labeling index and apoptotic index in recurrent astrocytic tumors. Pathol Oncol Res 7:267-278, 2001

29. Ridley AJ, Schwartz MA, Burridge K, Firtel RA, Ginsberg $\mathrm{MH}$, Borisy $\mathrm{G}$, et al: Cell migration: integrating signals from front to back. Science 302:1704-1709, 2003

30. Sanai N, Polley MY, McDermott MW, Parsa AT, Berger MS: An extent of resection threshold for newly diagnosed glioblastomas. J Neurosurg 115:3-8, 2011

31. Star EN, Kwiatkowski DJ, Murthy VN: Rapid turnover of actin in dendritic spines and its regulation by activity. Nat Neurosci 5:239-246, 2002

32. Stupp R, Hegi ME, Mason WP, van den Bent MJ, Taphoorn MJ, Janzer RC, et al: Effects of radiotherapy with concomitant and adjuvant temozolomide versus radiotherapy alone on survival in glioblastoma in a randomised phase III study: 5-year analysis of the EORTC-NCIC trial. Lancet Oncol 10:459-466, 2009

33. Stupp R, Mason WP, van den Bent MJ, Weller M, Fisher B, Taphoorn MJ, et al: Radiotherapy plus concomitant and adjuvant temozolomide for glioblastoma. $\mathbf{N}$ Engl J Med 352:987-996, 2005

34. Taiyab A, Rao ChM: HSP90 modulates actin dynamics: inhibition of HSP90 leads to decreased cell motility and impairs invasion. Biochim Biophys Acta 1813:213-221, 2011

35. Toschi L, Jänne PA: Single-agent and combination therapeutic strategies to inhibit hepatocyte growth factor/ MET signaling in cancer. Clin Cancer Res 14:5941-5946, 2008

36. Tsang DS, Khan L, Perry JR, Soliman H, Sahgal A, Keith JL, et al: Survival outcomes in elderly patients with glioblastoma. Clin Oncol (R Coll Radiol) 27:176-183, 2015

37. van den Bent MJ, Dubbink HJ, Marie Y, Brandes AA, Taphoorn MJ, Wesseling P, et al: IDH1 and IDH2 mutations 
are prognostic but not predictive for outcome in anaplastic oligodendroglial tumors: a report of the European Organization for Research and Treatment of Cancer Brain Tumor Group. Clin Cancer Res 16:1597-1604, 2010

38. Yan H, Parsons DW, Jin G, McLendon R, Rasheed BA, Yuan W, et al: IDH1 and IDH2 mutations in gliomas. N Engl J Med 360:765-773, 2009

39. Zhang C, Zhang J, Hao J, Shi Z, Wang Y, Han L, et al: High level of miR-221/222 confers increased cell invasion and poor prognosis in glioma. J Transl Med 10:119, 2012

\section{Disclosures}

The authors report no conflict of interest concerning the materials or methods used in this study or the findings specified in this paper.

\section{Author Contributions}

Conception and design: Wanibuchi, Ohtaki, Kataoka-Sasaki, Sasaki, Honmou. Acquisition of data: Wanibuchi, Ohtaki. Analysis and interpretation of data: Wanibuchi, Ohtaki, KataokaSasaki, Sasaki, Oka, Noshiro, Akiyama, Mikami, Mikuni, Honmou. Drafting the article: Wanibuchi, Ohtaki, Sasaki, Mikuni, Honmou. Critically revising the article: Wanibuchi, Ohtaki, Kataoka-Sasaki, Sasaki, Honmou. Approved the final version of the manuscript on behalf of all authors: Wanibuchi. Statistical analysis: Wanibuchi, Ohtaki, Kataoka-Sasaki, Oka, Noshiro. Administrative/technical/material support: Oka, Noshiro, Akiyama, Mikami, Mikuni, Kocsis, Honmou. Study supervision: Mikuni, Kocsis, Honmou.

\section{Correspondence}

Masahiko Wanibuchi, Department of Neurosurgery, Sapporo Medical University School of Medicine, South 1, West 16,

Chuo-ku, Sapporo, Hokkaido 060-8543, Japan. email: wanibuti@ sapmed .ac.jp. 